\title{
Aryl hydrocarbon receptor activation modulates $\gamma \delta$ intestinal intraepithelial lymphocytes and protects against ischemia/reperfusion injury in the murine small intestine
}

\author{
ZHICAO ZHANG, AIMIN PU, MIN YU, WEIDONG XIAO, LIHUA SUN, YUJIAO CAI and HUA YANG \\ Department of General Surgery, Xinqiao Hospital, Army Medical University, Chongqing 400037, P.R. China
}

Received April 23, 2018; Accepted November 26, 2018

DOI: $10.3892 / \mathrm{mmr} .2019 .9823$

\begin{abstract}
The pathogenesis of intestinal ischemia/reperfusion $(\mathrm{I} / \mathrm{R})$ is associated with dysregulation of the intestinal immune system. The aryl hydrocarbon receptor (AhR), a receptor expressed in gamma-delta $(\gamma \delta)$ intraepithelial lymphocytes (IELs), is thought to regulate inflammation in the bowel. $\gamma \delta$ IELs are a key immunologic compartment with a capacity to modulate immune responses. In the present study, the function of the AhR in $\gamma \delta I E L s$ in a mouse model of intestinal I/R injury was investigated to determine whether the AhR attenuates intestinal injury induced by intestinal I/R. Mice were assigned to three groups: sham, I/R and I/R+6-formylindolo(3,2-b) carbazole (FICZ). The sham group received no ischemia treatment, whereas the I/R and I/R+FICZ groups underwent upper mesenteric vessel ischemia for $30 \mathrm{~min}$. The I/R group was injected intraperitoneally with $0.3 \mathrm{ml}$ saline and the I/R+FICZ group was administered $1 \mu \mathrm{g}$ of FICZ before a subsequent $6 \mathrm{~h}$ reperfusion. Then, the mice were sacrificed and the entire small intestinal tissues were collected for histologic examination. The phenotype and apoptosis of $\gamma \delta$ IELs and activation of $\mathrm{CD}^{+}$and $\mathrm{CD}^{+}$IELs were examined using flow cytometry. The cytokine mRNA and anti-apoptosis gene expression in IELs were measured by qPCR. FICZ increased the $\gamma \delta I E L$

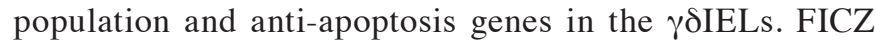
reduced the percentage of activated $\mathrm{CD}^{+}$and $\mathrm{CD} 8^{+}$subpopulations and the expression of pro-inflammatory mediator genes in IELs. FICZ inhibited inflammation in the gastrointestinal tract of mice with $\mathrm{I} / \mathrm{R}$ injury. These results suggest that the AhR plays an important role in protecting the small intestine from I/R and increasing the $\gamma \delta$ IEL population by decreasing apoptosis of $\gamma \delta$ IELs.
\end{abstract}

Correspondence to: Professor Hua Yang, Department of General Surgery, Xinqiao Hospital, Army Medical University, 183 Xinqiao Main Street, Chongqing 400037, P.R. China

E-mail: huayang@tmmu.edu.cn

Key words: ischemia-reperfusion injury, aryl hydrocarbon receptor, $\gamma \delta$ intestinal intraepithelial lymphocyte

\section{Introduction}

Intestinal ischemia/reperfusion (I/R), a common clinical event, is always related to medical conditions, such as serious trauma, hemorrhagic shock, vascular occlusion, or organ transplantation (1). I/R injury has been divided into an initial phase, which is due to the synthesis of oxygen-free radicals, followed by a later phase involving activation of the innate immune system (2). Ultimately, remote organ injury leads to systemic inflammatory response syndrome and multiple organ dysfunction syndrome. Therefore, interference with the activation or activity of the innate immune system may be beneficial for alleviation and prevention of I/R-induced intestinal damage.

6-Formylindolo(3,2-b)carbazole (FICZ) is a ligand of the aryl hydrocarbon receptor (AhR), which is part of the basic helix-loop-helix/Per-Arnt-Sim superfamily (3). Generally, the $\mathrm{AhR}$ is bound to several co-chaperones in an inactive form in the cytosol. Once bound by a ligand, the AhR translocates to the nucleus to initiate the transcription of a variety of genes (4). The best characterized target genes of the AhR include the cytochrome P450 family members, CYP1A1 and CYP1A2 (5). AhR signaling plays an important role in the control of several components of the innate immune system, including dendritic cells (DCs), macrophages, and innate lymphoid cells (ILCs) (6). Indeed, the AhR in macrophages limits lipopolysaccharide (LPS)-induced inflammation (7) and the AhR in DCs mediates the anti-inflammatory activities of lipoxin (8). Moreover, the AhR serves to induce expression of Bcl-2, c-Kit, IL-7R and Notch2, which together promote the survival of ILCs. Therefore, the AhR provides a molecular pathway by which the environment can affect the innate immune response and the development of innate immune-mediated injury.

Intestinal intraepithelial lymphocytes (IELs), which are involved in regulating mucosal immune responses, are essential intestinal innate immune cells. It has been shown that IELs not only maintain the intestinal epithelial barrier function and initiate the early response to infection in the intestine (9), but regulate activation of immune cells (10). Gamma-delta $(\gamma \delta)$ IELs constitute $30-50 \%$ of IELs in the small intestine (11). A role for $\gamma \delta I E L s$ has been reported in maintaining the protective barrier in DSS-treated mice and $\gamma \delta$ IELs are indispensable for restricting the mucosal penetration of commensal bacteria (12). It has also been shown that $\gamma \delta$ IELs contribute to mucosal homeostasis (13) by secreting keratinocyte growth 
factor (KGF) (14) and antimicrobial peptides (15) and by suppressing $\mathrm{CD}^{+} \mathrm{T}$ cell expansion through the production of TGF- $\beta$ and IL-10 (13). Our group has demonstrated that I/R-induced acute intestinal mucosal barrier damage significantly reduced the proportion of small intestine $\gamma \delta I E L s$ and that $\mathrm{I} / \mathrm{R}$ can trigger inflammatory responses and increase the apoptosis of IELs (16). Moreover, we also demonstrated that FICZ-mediated AhR activation inhibits inflammation in the gastrointestinal tract in mice with DSS-induced colitis (17). The AhR is highly expressed in $\gamma \delta$ IELs and has been reported to function in $\gamma \delta$ IELs. Mice without the AhR exhibit a $>95 \%$ loss of $\gamma \delta$ IELs in the small intestine (18). We also demonstrated that administration of FICZ increases the proportion of $\gamma \delta$ IELs and downregulates inflammatory responses in colitis. In the present study, we investigated the effect of FICZ on changes in $\gamma \delta$ IELs and on the subsequent inflammatory response to $I / R$ injury of the small intestine. Indeed, AhR activation may be a crucial factor for preventing I/R-induced intestinal mucosal injury.

\section{Materials and methods}

Animal experiments. Male C57BL/6 mice weighing $20 \pm 2 \mathrm{~g}$ were purchased from the Laboratory Animal Center of the Army Medical University (Chongqing, China). All animals were 6-8 weeks of age and specific pathogen-free. At the beginning of the study, the animals were housed under temperature-, humidity-, and light-controlled conditions. The laboratory animals were cared for according to the Guidelines for the Care and Use of Laboratory Animals, as set forth and approved by the University Committee at the Army Medical University.

Operative procedures. After a 12-h fast, the mice were anesthetized with an intraperitoneal injection of sodium pentobarbital $(50 \mathrm{ml} / \mathrm{kg})$. The abdomens were opened via midline incisions. The mice were randomly assigned to the sham group and two I/R groups, as follows: i) sham group, no intestinal ischemia treatment; ii) I/R group, the superior mesenteric vessels (SMVs) were occluded for $30 \mathrm{~min}$ and an intraperitoneal injection of saline $(0.3 \mathrm{ml})$ was administered $6 \mathrm{~h}$ before reperfusion; and iii) I/R+FICZ group, an intraperitoneal injection of FICZ $[1 \mu \mathrm{g} /$ mouse $(0.3 \mathrm{ml})]$ was given before reperfusion. Then, the mice in the three groups were sacrificed by cervical dislocation. The mice were sprayed with $70 \%$ ethanol, an abdominal midline incision was performed and then the mesenteric lymph nodes were isolated and collected from the entire small intestine. The same individual performed these manipulations. The experiment was repeated at least three times.

Histologic examination. The same intestinal segment $(2 \mathrm{~cm})$ from each animal was removed and immediately placed in $4 \%$ paraformaldehyde. Paraffin blocks were prepared from the tissue pieces. The blocks were cut into sections (5- $\mu \mathrm{m}$ thick), stained with hematoxylin and eosin (H\&E) and observed by light microscopy. Histologic evaluations were graded from 0-5 by a single observer, as follows: grade 0 , normal morphology; grade 1, sub-epithelial edema and partial separation of apical cells; grade 2, moderate lifting of enterocytes from the tips of the villi; grade 3 , lifting of enterocytes from both the tips and sides of the villi, including the superficial crypts; grade 4, partial mucosal necrosis of the lamina propria; and grade 5, total mucosal necrosis.

Detection of intestinal permeability. After the small intestine was harvested, a 3-cm section of the Peyer's patch-free jejunum along the mesenteric border was opened and gently washed three times with cool RPMI-1640 medium to cleanse the luminal contents. The full-thickness small intestine was fixed in a modified Ussing chamber (Physiologic Instruments, San Diego, CA, USA). Each half cell (mucosal and serosal) was filled with $5 \mathrm{ml}$ of pre-heated $37^{\circ} \mathrm{C}$ Krebs-Ringer solution buffer containing $110.0 \mathrm{mM} \mathrm{NaCl}, 5.5 \mathrm{mM} \mathrm{KCl}, 3.0 \mathrm{mM}$ $\mathrm{CaCl}_{2}, 1.2 \mathrm{mM} \mathrm{MgCl}_{2}, 1.4 \mathrm{mM} \mathrm{KH} \mathrm{PO}_{4}$ and $29.0 \mathrm{mM}$ $\mathrm{NaHCO}_{3}$, adjusted to $\mathrm{pH}$ 7.4. The cells were continuously oxygenated with $\mathrm{O}_{2} / \mathrm{CO}_{2}(95 \% / 5 \%)$ and stirred by gas flow in the chambers. One pair of $\mathrm{Ag} / \mathrm{AgCl}$ electrodes (Physiologic Instruments) with $3 \mathrm{mM} \mathrm{KCl}$ in $3 \%$ agar bridges was used to calculate the transepithelial potential difference and another pair of Pt electrodes was used for current passage. The transmembrane resistance (TER) was determined using Ohm's law. The baseline short circuit current (ISC) was recorded for up to $120 \mathrm{~min}$ after a 20 -min equilibration period.

IEL isolation. The entire small intestine was removed and placed in tissue culture media (RPMI-1640). The small intestine was cut longitudinally into 5 -mm pieces, washed 3 times with the same tissue culture media, and incubated in isolation buffer (54 ml of PBS, $22.8 \mathrm{mg}$ of EDTA, $9.6 \mathrm{mg}$ of DTT, and $6 \mathrm{ml}$ of $10 \%$ fetal calf serum) with continuous gentle shaking at $37^{\circ} \mathrm{C}$ for $30 \mathrm{~min}$. After centrifugation, the pellets were purified in $40 \%$ isotonic Percoll (GE Healthcare Biosciences) and reconstituted in RPMI-1640 tissue culture media. The viability of the IELs exceeded $96 \%$ based on trypan blue exclusion staining.

Quantitative PCR ( $q P C R$ ). Total RNA was isolated from the sorted IELs using Trizol reagent (Invitrogen; Thermo Fisher Scientific, Inc., Waltham, MA, USA) according to the manufacturer's instructions. Briefly, RNA was reverse-transcribed into complementary DNA (cDNA) using a SuperScript First-Strand Synthesis System RT-PCR kit (Invitrogen; Thermo Fisher Scientific, Inc.), and this cDNA was used as a template for the amplification of $\beta$-actin, TNF- $\alpha$, IL- $1 \beta$, IL-6, Bcl2, Bcl211, KGF, CYP1A1 and CD127. The primer sequences are presented in Table I (gene symbol: forward, reverse). Gene expression was determined by qPCR, which was performed using a SYBR PrimeScript RT kit, as described by the manufacturer (Takara Bio Inc., Kusatsu, Japan). The PCR mixture (20 $\mu$ l final volume per reaction) was prepared per the manufacturer's protocol. Amplifications were performed under the following conditions on a 7500 Real-Time PCR system (Applied Biosystems; Thermo Fisher Scientific, Inc.): $94^{\circ} \mathrm{C}$ for $5 \mathrm{~min} ; 35$ cycles of $94^{\circ} \mathrm{C}$ for $30 \mathrm{sec}, 59^{\circ} \mathrm{C}$ for $30 \mathrm{sec}$, and $72^{\circ} \mathrm{C}$ for $30 \mathrm{sec}$; and $72^{\circ} \mathrm{C}$ for $10 \mathrm{~min}$. The expression of each target gene was calculated by the $2^{-\Delta \Delta C q}$ method (19).

Flow cytometric analysis. The IEL subtype was confirmed by fluorescent antibody staining, which was detected using flow cytometry. The following anti-mouse monoclonal 
Table I. Primer sequences used for qPCR assays of inflammatory mediators.

\begin{tabular}{|c|c|}
\hline Gene name & 5'-3' primer sequence \\
\hline \multirow[t]{2}{*}{$\beta$-actin } & F: CTTCTTTGCAGCTCCTTCGTT \\
\hline & R: AGGAGTCCTTCTGACCCATTC \\
\hline \multirow[t]{2}{*}{$T N F-\alpha$} & F: TCTTCTCATTCCTGCTTGTGG \\
\hline & R: CACTTGGTGGTTTGCTACGAC \\
\hline \multirow[t]{2}{*}{$I L-1 \beta$} & F: CCAGCTTCAAATCTCACAGCAG \\
\hline & R:CTTCTTTGGGTATTGCTTGGGATC \\
\hline \multirow[t]{2}{*}{ IL-6 } & F: TCCAGTTGCCTTCTTGGGAC \\
\hline & R: GTACTCCAGAAGACCAGAGG \\
\hline \multirow[t]{2}{*}{$B c l 2$} & F: AGTACCTGAACCGGCATCTG \\
\hline & R: AGGTATGCACCCAGAGTGATG \\
\hline \multirow[t]{2}{*}{ Bcl2ll } & F: ATGACCACCTAGAGCCTTTGGA \\
\hline & R: GAAGAGTGAGCCCAGCAGAAC \\
\hline \multirow[t]{2}{*}{$K G F$} & F: CGCAAATGGATACTGACACG \\
\hline & R: GGGCTGGAACAGTTCACACT \\
\hline \multirow[t]{2}{*}{ CYPIA1 } & F: CCAAGAGCTGCTCAGCATAG \\
\hline & R: GGCATCCAGGGAAGAGTTAG \\
\hline \multirow[t]{2}{*}{$C D 127$} & F: GCGGACGATCTCCTTCTG \\
\hline & R: AGCCCCACATATTTGAAATTCCA \\
\hline
\end{tabular}

$T N F-\alpha$, tumor necrosis factor- $\alpha ; I L-1 \beta$, interleukin- $1 \beta ; I L-6$, interleukin-6; Bcl2, B-cell lymphoma 2; Bcl2l1, BCL2-like 1; KGF, keratinocyte growth factor; CYP1A1, cytochrome P450 1A1; CD127, cluster of differentiation 127 ; F, forward; $R$, reverse.

antibodies (eBioscience, Inc., San Diego, CA, USA) were used: CD45-PE (dilution 1:100; cat. no. 12-0451-82); CD45-PerCP-Cy5.5 (dilution 1:100; cat. no. 45-0451-82); CD4-PE (dilution 1:100; cat. no. 12-0041-82); CD69-FITC (dilution 1:100; cat. no. 11-0691-82); CD8 $\alpha$-APC (dilution 1:100; cat. no. 17-0081-82); CD8 $\beta$-PE (dilution 1:100; cat. no. 12-0083-82); TCR $\beta$-APC (dilution 1:100; cat. no. 17-5961-82); CD127-PE-CY (dilution 1:100; cat. no. 25-1271-82); and TCR $\gamma \delta$-FITC (dilution 1:100; cat. no. 11-5711-82). Isotype-matched, irrelevant antibodies (eBioscience, Inc.) were used as negative controls: Rat IgG2b kappa Isotype Control (eB149/10H5), PE (cat. no. 12-4031-82); Rat IgG2b kappa Isotype Control (eB149/10H5), PerCP-Cyanine5.5 (cat. no. 45-4031-80); Armenian Hamster IgG Isotype Control (eBio299Arm), FITC (cat. no. 11-4888-81); Rat IgG2a kappa Isotype Control (eBR2a), APC (cat. no. 17-4321-81). The apoptotic ratios of $\gamma \delta$ IELs among the different groups were detected by flow cytometry per the manufacturer's protocol. $\gamma \delta$ IELs were stained with PE-Annexin V and cellular DNA was stained with7-aminoactinomycin D (7-AAD), as described in the protocol. The acquisition and analysis were performed using MoFlo XDP (Beckman Coulter, Inc., Brea, CA, USA).

Total $\gamma \delta I E L$ isolation. To study associated gene expression in $\gamma \delta$ IELs, total IELs were stained with the following anti-mouse monoclonal antibodies (eBioscience, Inc.): FITC anti-mouse TCR $\gamma \delta$ (dilution 1:100; cat. no. 11-5711-82); and $\mathrm{PE}$ anti-mouse CD8 $\beta$ (dilution 1:100; cat. no. 12-0083-82). $\gamma \delta$ IELs were purified using a cell sorter (Beckman Coulter). The $\mathrm{TCR}^{+} / \mathrm{CD} 8 \beta^{-}$subsets were isolated from $\gamma \delta$ IELs and the purity was $\geq 98 \%$.

Statistical analysis. All data are presented as the mean \pm standard deviation (SD). The results were analyzed by one-way analysis of variance (ANOVA) followed by Tukey's post-hoc test using SPSS statistical software package. A $\mathrm{P}$-value $<0.05$ was considered to indicate statistical significance.

\section{Results}

$I / R$ induces changes in intestinal morphology and FICZ ameliorates inflammation following intestinal I/R injury. Based on our previous study, the destruction of intestinal morphology was more severe $6 \mathrm{~h}$ after intestinal I/R. Therefore, this time-point was chosen for subsequent experiments. H\&E was used to stain the small intestines from mice in the sham, $\mathrm{I} / \mathrm{R}$ and I/R+FICZ groups. Representative images from each group are presented in Fig. 1A. The intestines of the sham group showed an intact epithelium, a normal villous length, well-defined glands, and minimal leukocyte infiltration in the mucosa. In contrast, severe mucosal damage, including villous blunting, epithelial denudation, gland distortion, leukocyte inflammation, and lamina propria disintegration, were observed in the I/R group (Fig. 1B). FICZ attenuated these changes. The histologic scores of intestinal mucosal injury are presented as the mean \pm SD (Fig. 1D). Moreover, changes in intestinal permeability were detected using Ussing chambers. Compared with the I/R+FICZ group, the TER of the I/R group was significantly lower $(\mathrm{P}<0.01$; Fig. $1 \mathrm{C})$. These results showed that FICZ attenuates the IR-induced destruction of intestinal morphology and intestinal barrier function.

FICZ downregulates pro-inflammatory mediator mRNA expression in small intestinal IELs. During reperfusion injury, pro-inflammatory molecules, such as IL-1 $\beta$, IL- 6 and TNF- $\alpha$, directly induce tissue damage to affect intestinal barrier function and are potent activators of other neutrophils. Based on the attenuation of decreased intestinal barrier function, the effect of FICZ was confirmed on IL-1 $\beta$, IL- 6 and TNF- $\alpha$ gene expression. The expression of IL-1 $\beta$, IL- 6 and TNF- $\alpha$ mRNA in intestinal IELs is shown. Real-time PCR showed that IEL-derived IL-1 $\beta$ ( $\mathrm{P}<0.05$; Fig. 2), IL-6 ( $\mathrm{P}<0.05$; Fig. 2), and TNF- $\alpha(\mathrm{P}<0.01$; Fig. 2) mRNA expression was significantly increased after I/R compared with the sham group. Importantly, the expression of IL-1 $\beta(\mathrm{P}<0.05 ;$ Fig. 2$)$, IL-6 (P<0.05; Fig. 2$)$ and TNF- $\alpha(\mathrm{P}<0.01$; Fig. 2) was significantly decreased in the I/R+FICZ group when compared with the I/R group.

FICZ reduces the percentage of activated $\mathrm{CD}^{+}{ }^{+}$and $\mathrm{CD} 8^{+}$ IEL subpopulations. CD69, an early T cell activation marker, is expressed in most IELs, indicating the intensity of inflammatory responses. In the present study, CD69 was used to assess $\mathrm{CD} 4^{+}$and $\mathrm{CD} 8^{+}$IEL subsets. Compared with the sham group, $\mathrm{CD} 4^{+} \mathrm{CD} 69^{+}$and $\mathrm{CD} 8^{+} \mathrm{CD} 69^{+}$IEL subpopulations were significantly increased in the $\mathrm{I} / \mathrm{R}$ group $(55.30 \pm 1.92 \%$ vs. $49.40 \pm 2.22 \%, \mathrm{P}<0.05 ; 85.60 \pm 3.22 \%$ vs. $77.40 \pm 2.00 \%, \mathrm{P}<0.05$; Fig. 3). Notably, the I/R+FICZ group had a decreased population of IELs compared with the I/R group $(43.60 \pm 2.90 \%$ vs. 
A

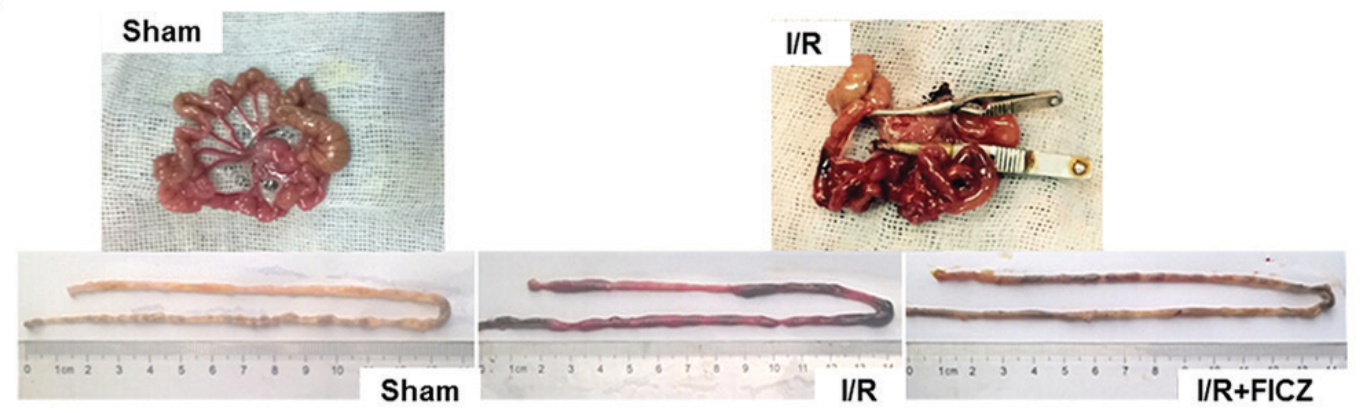

B

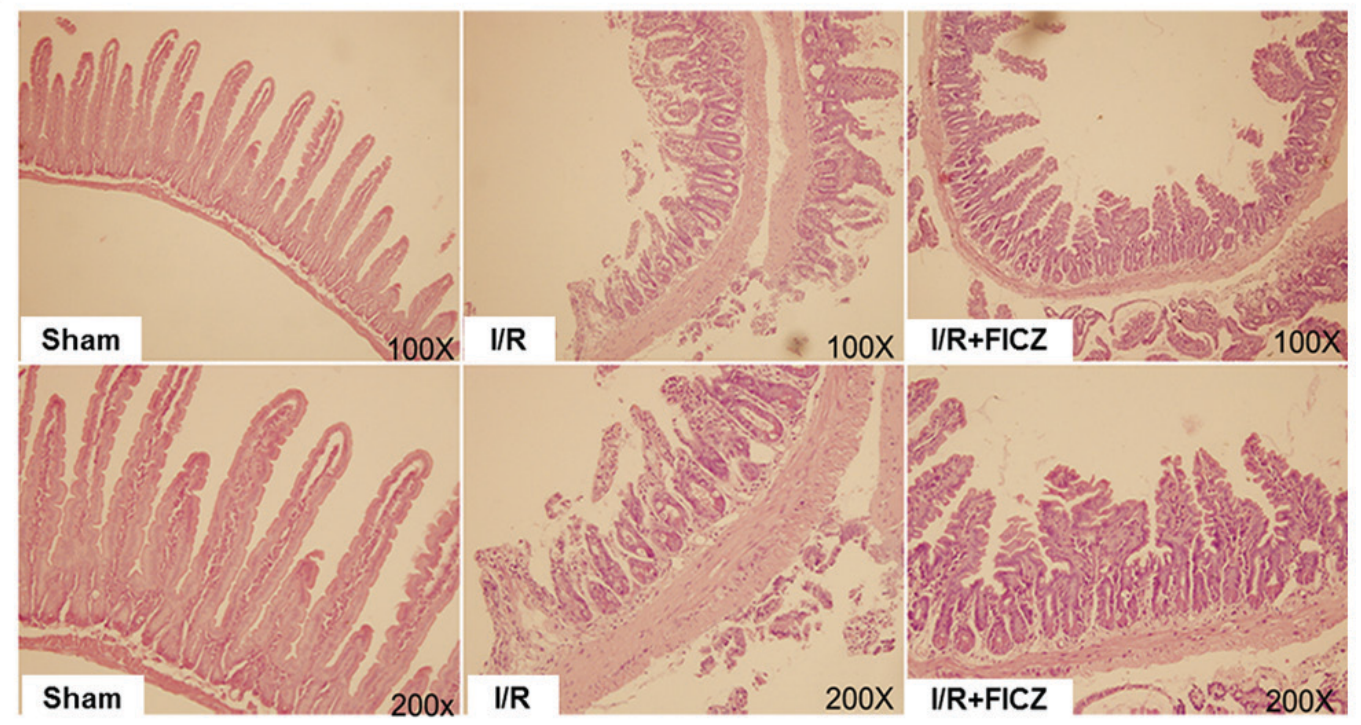

C

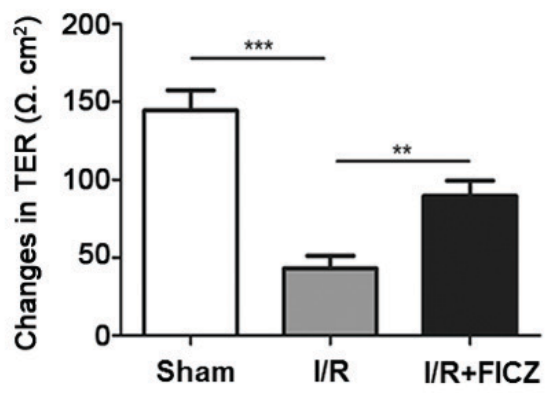

D

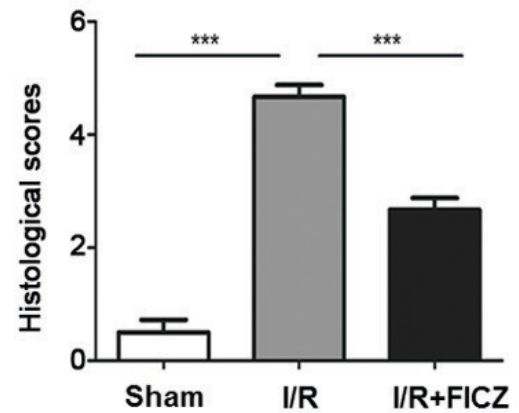

Figure 1. FICZ ameliorates I/R-induced dysfunction of the small intestine. (A) The mice were sacrificed $6 \mathrm{~h}$ after reperfusion, and gut tissue samples were obtained. (B) Representative intestinal sections from sham-, I/R-, and I/R+FICZ-treated WT mice were stained with hematoxylin and eosin (x100 and x200 magnification). (C) Histologic scores of intestinal mucosal injury are presented as the mean \pm SD, which were determined as described in Materials and methods. (D) The permeability of the small intestine was evaluated based on the TER. Each group had 2-3 mice and every experiment was repeated three times. ${ }^{* *} \mathrm{P}<0.01 ;{ }^{* * * *} \mathrm{P}<0.001$. I/R, ischemia/reperfusion; FICZ, 6-formylindolo(3,2-b)carbazole; TER, transmembrane resistance.

$55.30 \pm 1.92 \%, \mathrm{P}<0.01 ; 72.10 \pm 2.86 \%$ vs. $85.60 \pm 3.22 \%, \mathrm{P}<0.01$ Fig. 3).

FICZ enhances the percentage of $T C R \gamma \delta^{+} / C D 45^{+}$and $C D 127^{+} / T C R \gamma \delta^{+} T$ cells and decreases apoptosis in $\gamma \delta I E L s$. Reductions in intestinal inflammation suggested that FICZ may alter IEL phenotypes. Thus, a series of phenotypic analyses were performed by flow cytometry. The T-cell receptor (TCR) subpopulations were examined. Compared with the sham group, intestinal I/R treatment resulted in lower percentages of small intestinal IEL $\mathrm{TCR} \gamma \delta^{+} / \mathrm{CD} 45^{+} \mathrm{T}$ cells $(29.20 \pm 2.43 \%$ vs. $51.30 \pm 1.87 \%, \mathrm{P}<0.001$; Fig. $4 \mathrm{~A})$ and $\mathrm{CD} 127^{+} / \mathrm{TCR} \gamma \delta^{+} \mathrm{T}$ cells $(2.31 \pm 0.26 \%$ vs. $4.65 \pm 0.32 \%, \mathrm{P}<0.05$; Fig. 4B). The percentage of $\mathrm{TCR} \gamma \delta^{+} / \mathrm{CD}^{2} 5^{+} \mathrm{T}$ cells $(40.80 \pm .72 \%$ vs. $29.20 \pm 2.43 \%$, $\mathrm{P}<0.01$; Fig. $4 \mathrm{~A})$ and $\mathrm{CD} 127^{+} / \mathrm{TCR} \gamma \delta^{+} \mathrm{T}$ cells $(4.82 \pm 0.50 \%$ vs. $2.31 \pm 0.26 \%, \mathrm{P}<0.01$; Fig. 4B) among small intestinal IELs was significantly higher in the I/R+FICZ group than the I/R group. Considering the decreased percentage of TCR $\gamma \delta$ cells, the survival of $\gamma \delta$ IELs was subsequently investigated by assaying I/R apoptosis using flow cytometry. There were significant 


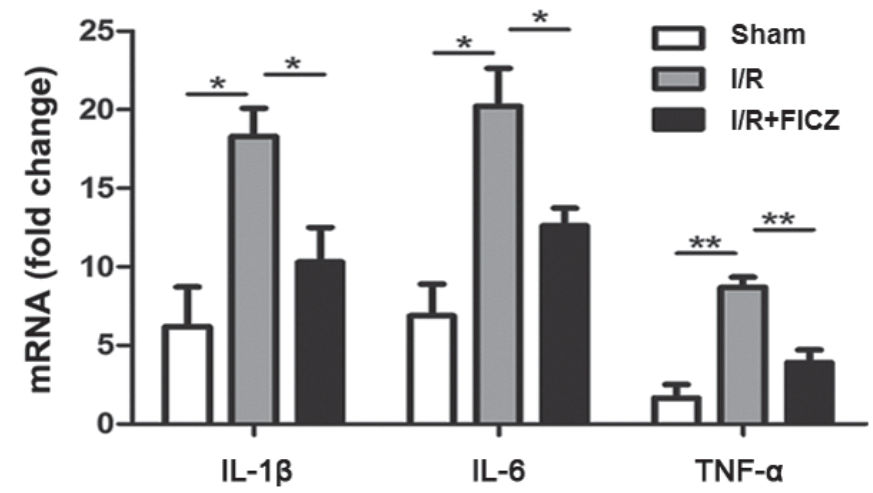

Figure 2. FICZ downregulates the expression of pro-inflammatory mediator genes in small intestinal IEL cells. Expression of IL-1 $\beta$, IL- 6 and TNF- $\alpha$ genes in IELs was analyzed by qPCR. Each group had 2-3 mice and every experiment was repeated three times. ${ }^{*} \mathrm{P}<0.05$; ${ }^{* *} \mathrm{P}<0.01$. FICZ, 6-formylindolo(3,2-b)carbazole; IEL, intraepithelial lymphocyte.

changes in the apoptosis of these cells. Compared with the sham group, the apoptosis rate (early and late) of $\gamma \delta$ IELs was significantly increased after I/R $(3.26 \pm 0.51 \%$ vs. $0.78 \pm 0.23 \%$, $\mathrm{P}<0.01$; Fig. 4C). FICZ administration significantly attenuated apoptosis induced by intestinal I/R $(1.53 \pm 0.33 \%$ vs. $3.26 \pm 0.51 \%, \mathrm{P}<0.05$; Fig. 4C).

CYP1A1, KGF, Bcl2, Bcl2l1 and CD127 expression in small intestinal $\gamma \delta I E L s$. The expression of CD127, which binds to IL-7, is regulated throughout T cell development, activation, and survival, and CD127 signals are thus required for $\gamma \delta$ T cell development and survival. Since the proportion of $\mathrm{TCR} \gamma \delta^{+} / \mathrm{CD} 45^{+}$ $T$ cells was decreased after $I / R$, it was determined whether or not the expression of CD127 mRNA in small intestinal $\gamma \delta I E L s$ was altered. CD127 gene expression was significantly lower in the $I / R$ group than that noted in the sham group $(\mathrm{P}<0.05$; Fig. 5B). Bcl2 and Bcl211 expression is maintained by IL-7R signaling. Thus, we detected the expression of $\mathrm{Bcl} 2$ and $\mathrm{Bcl} 211$ mRNA in small intestinal $\gamma \delta I E L s$. The expression of $\mathrm{Bcl} 2$ and Bcl211 was significantly decreased after I/R ( $\mathrm{P}<0.05$; Fig. 5B). $\mathrm{KGF}$, which plays a critical role in intestinal epithelial growth and maintenance, is only expressed by $\gamma \delta$ IELs. Intestinal I/R resulted in a significant decrease in the expression of KGF mRNA $(\mathrm{P}<0.05$; Fig. 5A). As a standard indicator of AhR activation, the expression of CYP1A1 mRNA in $\gamma \delta$ IELs was significantly decreased after I/R when compared with the sham group $(\mathrm{P}<0.01$; Fig. 5A). Interestingly, the I/R+FICZ group had significantly higher CYP1A1expression when compared with the I/R group ( $\mathrm{P}<0.01$; Fig. $5 \mathrm{~A})$. $\mathrm{Bcl} 2$ and $\mathrm{Bcl} 211$ gene expression in the I/R+FICZ group increased significantly compared with the I/R group $(\mathrm{P}<0.05$; Fig. $5 \mathrm{~B})$. Moreover, compared with the I/R group, CD127 expression was significantly increased after FICZ treatment $(\mathrm{P}<0.01$; Fig. 5B). As expected, KGF gene expression was increased after FICZ administration compared with I/R alone ( $\mathrm{P}<0.01$; Fig. $5 \mathrm{~A})$.

\section{Discussion}

Intestinal ischemia/reperfusion (I/R) injury that occurs in a variety of clinical conditions may lead to local or systemic inflammatory responses $(10,20)$. As a source of pro-inflammatory stimuli and an important barrier against intestinal bacteria, the small intestine is vulnerable during local I/R (21). Intestinal I/R is considered an abdominal emergency and is associated with high morbidity and mortality rates. It has been reported that intestinal I/R injuries feature acute inflammation, oxygen radicals, and cytolysis in the intestine (22). Furthermore, the present study showed that the expression of inflammatory-associated mediator genes was increased in the small intestine, thus indicating the induction of an inflammatory response in the small intestine in response to $\mathrm{I} / \mathrm{R}$.

Intraepithelial lymphocytes (IELs) play an important role in maintaining the intestinal barrier function. Previous studies from our group have shown that the changes in IEL subset markers and functions are significant under some pathologic circumstances in the intestine (16). Moreover, there is a crucial positive correlation between $\mathrm{CD} 3^{+} \mathrm{CD} 8^{+} \mathrm{IELs}$ and disease activity in patients with ulcerative colitis (23). In a previous study, it was shown that TPN significantly affects the $\mathrm{CD} 4{ }^{+} \mathrm{CD} 8{ }^{-}$and $\mathrm{CD}^{+}{ }^{+} \mathrm{CD} 8^{+}$IEL subpopulations and induces loss of CD8 $\alpha \beta^{+}$IELs (16). Furthermore, intestinal I/R significantly reduced the CD8 $\alpha \alpha^{+}$subpopulation and increased the CD8a $\beta^{+}$subpopulation (16). CD69 expression is an index of $\mathrm{T}$ cell function (24), and CD69 expression might reflect the activated cytotoxic nature of IELs (25). $\mathrm{CD}^{+}{ }^{+} \mathrm{CD} 69^{+}$and $\mathrm{CD}^{+} \mathrm{CD} 69^{+}$co-staining is used to predict immune reactivity (26). This study showed that $\mathrm{CD}^{+}$and $\mathrm{CD}^{+}$had much higher percentages of CD69, which is consistent with the previous findings by our group in a DSS-induced mouse model (17). Treatment with FICZ decreased the percentage of the CD69 subpopulation, which could indicate attenuation of an inflammatory response. KGF is secreted by activated small intestinal $\gamma \delta$ IELs and this mitogenic growth factor plays an important role in restoring or maintaining the integrity of epithelial tissues after I/R injury (27). In this study, it was demonstrated that expression of KGF mRNA in $\gamma \delta$ IELs was downregulated in mice with intestinal I/R, suggesting that $\gamma \delta$ IELs are affected by I/R-induced small intestine injury, and as expected, FICZ significantly increased expression of KGF mRNA expression in the small intestine of mice after I/R.

The IL-7 receptor is composed of an $\alpha$-chain (CD127) and a $\beta$-chain (CD132). The expression of CD127, which binds to IL-7, is strictly regulated throughout T cell development, activation and survival. Therefore, its signals are required for $\gamma \delta$ T cell development and survival (28). IL-7 secreted by intestinal epithelial cells (IECs) directly binds to IL-7R, and this interaction maintains the survival of $\gamma \delta$ IELs. Many previous studies in animals have shown that the IL-7/IL-7R-dependent signaling pathway plays an important role in attenuating the inflammatory response in the intestinal mucosa and that $\gamma \delta$ IELs are absent when IL-7 and IL-7R genes are inactivated (29). Moreover, a previous study from our group showed that IL-7 expression in the small intestine is increased after I/R (30). In the present study, it was found that the proportion of $\gamma \delta$ IELs was decreased, and lower CD127 expression was detected on $\gamma \delta I E L s$ after small intestine I/R. The decrease in CD127 may be the key event that results in a decreased number of $\gamma \delta$ IELs. Importantly, intraperitoneal treatment with FICZ prior to reperfusion increased CD127 expression on $\gamma \delta$ IELs. 


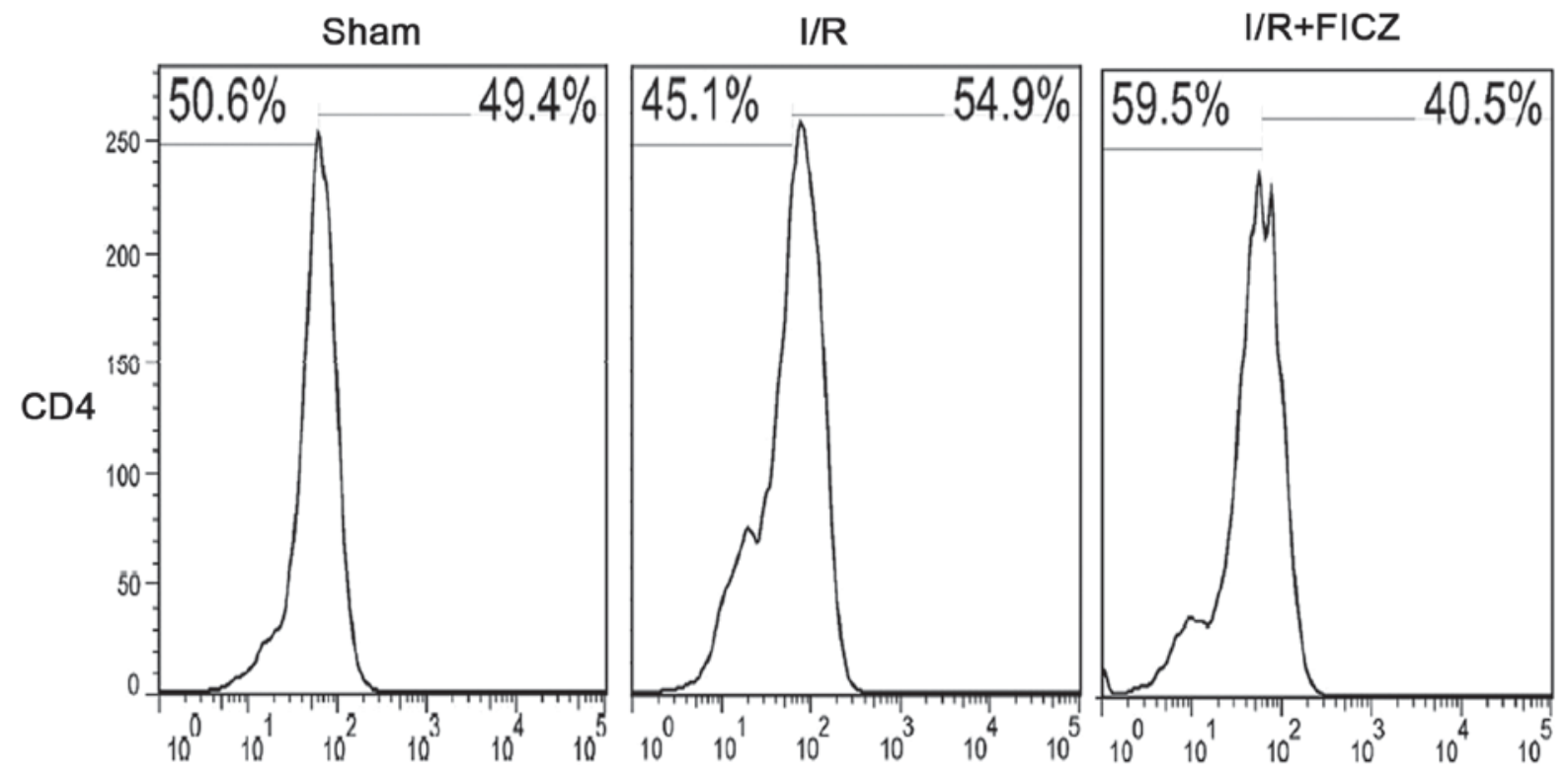

CD69
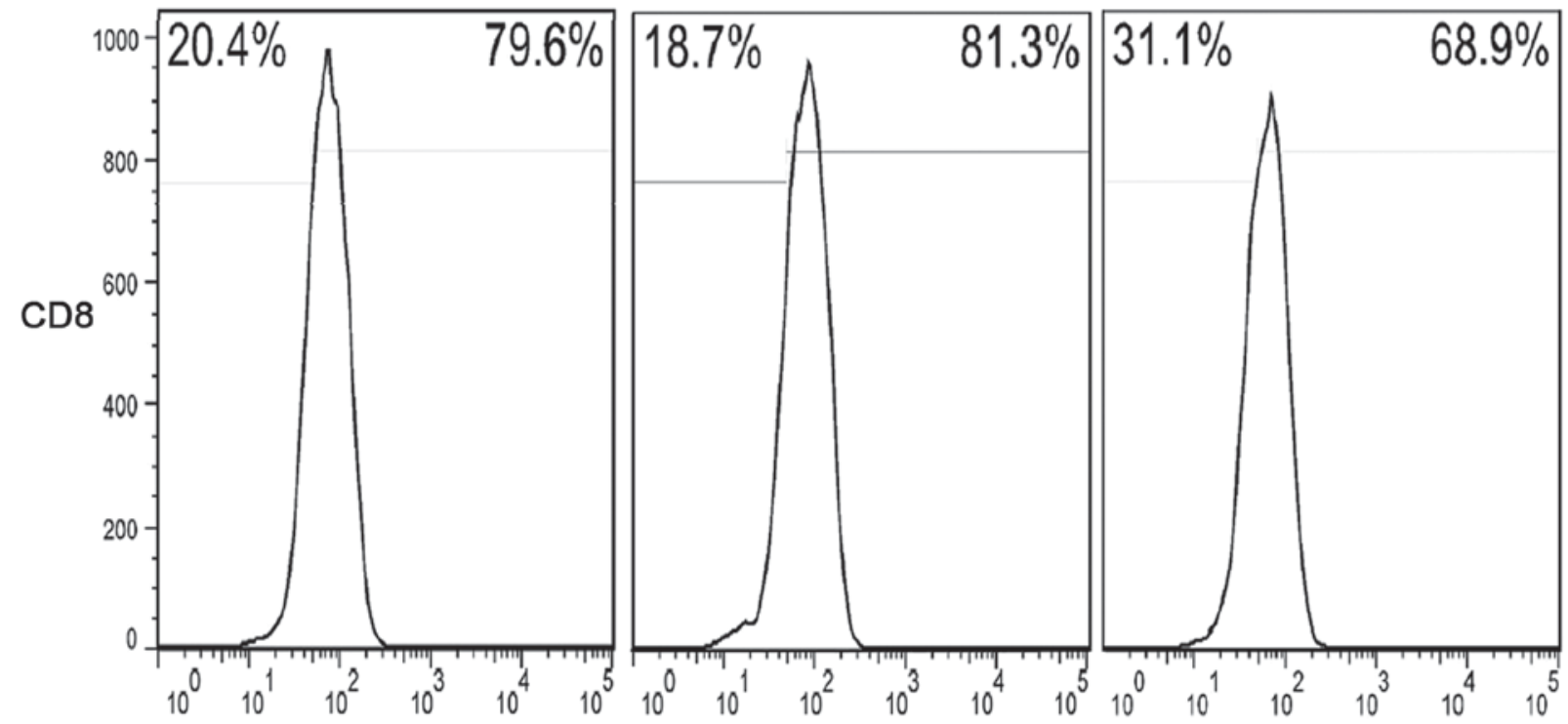

CD69

Figure 3. FICZ reduces the percentage of activated CD4 ${ }^{+}$and CD8 $8^{+}$IEL sub-populations. The percentage of activated CD4 ${ }^{+}$and $\mathrm{CD} 8^{+}$IEL subpopulations was analyzed by flow cytometry. Data were gated on $\mathrm{CD} 8^{+}$and $\mathrm{CD} 4^{+}$cells. Each group had 2-3 mice and every experiment was repeated three times. FICZ, 6-formylindolo(3,2-b)carbazole; IEL, intraepithelial lymphocyte. Data shown in the figure is derived from one presentative group.

IELs consist of TCR $\alpha \beta$ and TCR $\gamma \delta \mathrm{T}$ cells, and several studies have suggested that these subpopulations of IELs play a crucial role in modulating inflammatory diseases in the intestine $(31,32)$; however, consistent data from our group have shown that the population of $\alpha \beta$ IELs is always positively correlated with the intensity of the inflammatory response, but $\gamma \delta$ IELs have yielded contrary results $(16,17)$. Consistent with a previous study (33), small intestinal I/R increased the apoptosis of $\gamma \delta I E L s$. Whether or not the stable population of $\gamma \delta I E L s$ deterred the progressive inflammatory response remains unknown. Notably, our results showed that FICZ attenuated apoptosis and the inflammatory response, which explained why it is so important to sustain survival of $\gamma \delta$ IELs. These changes might partly explain why FICZ can ameliorate IR-induced acute inflammation of the intestine. Bcl-xL, also known as Bcl211, is a known inhibitor of apoptosis, the expression of which sustains the survival of $\mathrm{CD}^{+} \mathrm{T}$ cells (34). In the present study, increased expression of CD127 and Bcl-xL was detected after administration of FICZ, indicating that FICZ-activated AhR attenuated the decreased expression of CD127 and Bcl-xL in $\gamma \delta I E L s$ after I/R.

In the present study, severe epithelial damage was observed $6 \mathrm{~h}$ after intestinal I/R. The histologic features in the I/R group were consistent with previous studies on intestinal $\mathrm{I} / \mathrm{R}$ in animals $(35,36)$. Destruction of the epithelium 
A
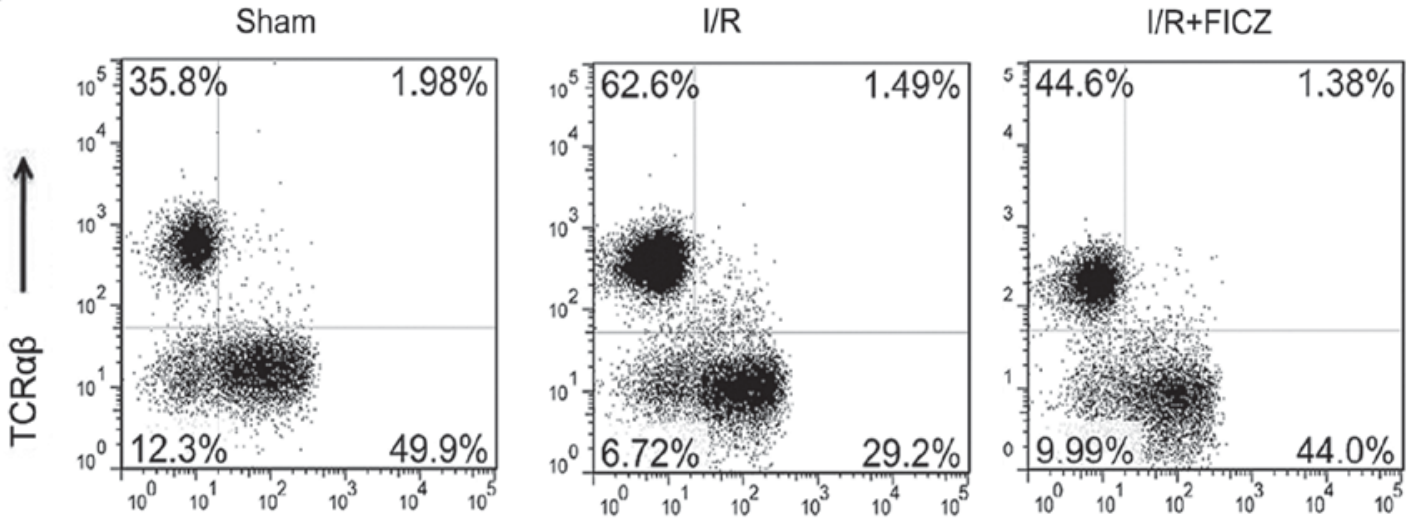

TCRyס

B
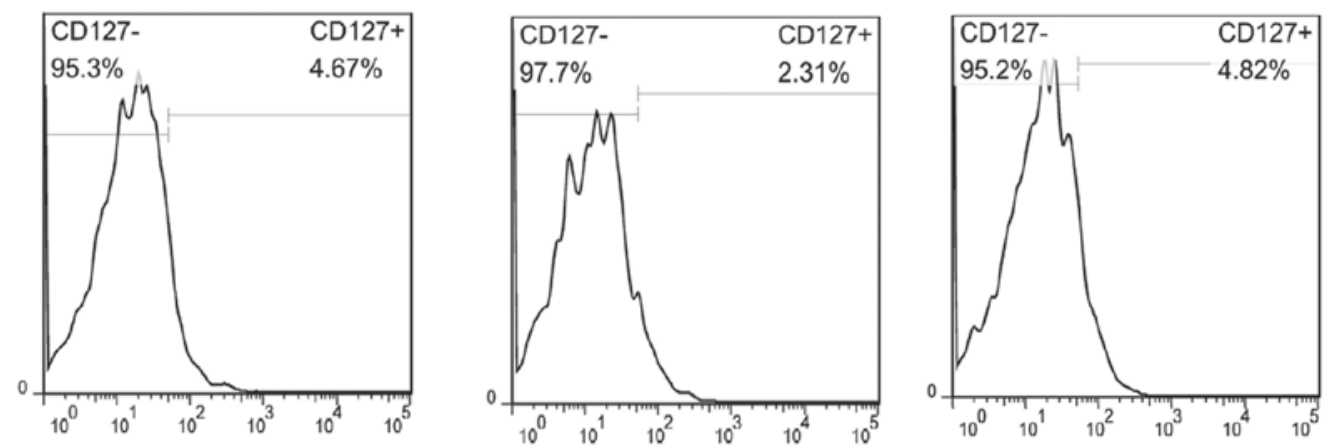

CD127

\section{C}
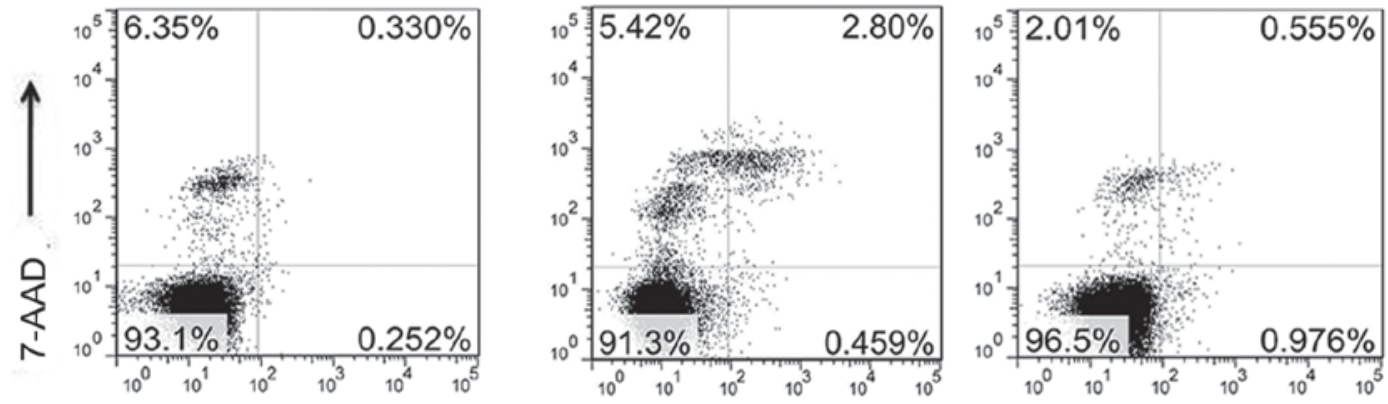

Yठ̄IEL

Annexin V-PE

Figure 4. FICZ alters IEL phenotypes and the apoptosis of $\gamma \delta$ IELs in the murine small intestine. (A) The percentage of TCR $\alpha \beta$ and TCR $\gamma \delta$ was analyzed by flow cytometry. (B) CD $127^{+}$IELs were analyzed in the $\gamma \delta$ IELs by flow cytometry. (C) Apoptosis of $\gamma \delta$ IELs was analyzed by flow cytometry. Each group had 2-3 mice and every experiment was repeated three times. FICZ, 6-formylindolo(3,2-b)carbazole; IELs, intraepithelial lymphocytes; TCR, T-cell receptor. Data shown in the figure is derived from one presentative group.

is often accompanied by increased permeability in the damaged mucosa (16,37). Consistent with previous results on morphology and physiology, this study showed that intestinal mucosal damage was less severe in the I/R+FICZ group. Therefore, AhR activation results in a greater restoration and higher anti-infection-associated gene expression, thus attenuating the severity of small intestinal epithelial injury. Based on these findings, FICZ, in ameliorating IR-induced small intestinal epithelial injury, could be involved in a complex mechanism; however, modulating the changes in $\gamma \delta$ IEL gene expression may be one of several crucial contributory mechanisms. A previous study by our group showed that intraperitoneal FICZ injection increased the percentage of the $\gamma \delta$ IEL subset in mice with DSS-induced colitis (17). It 
A

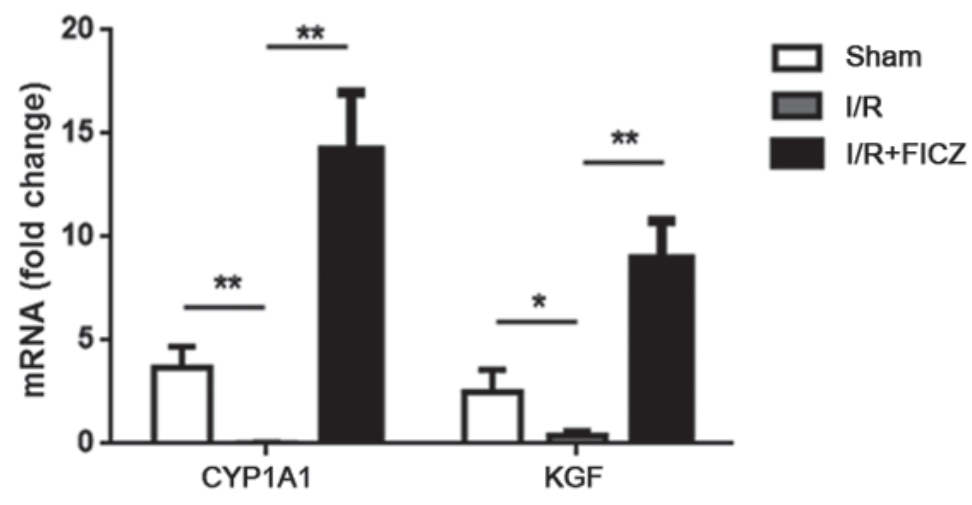

B

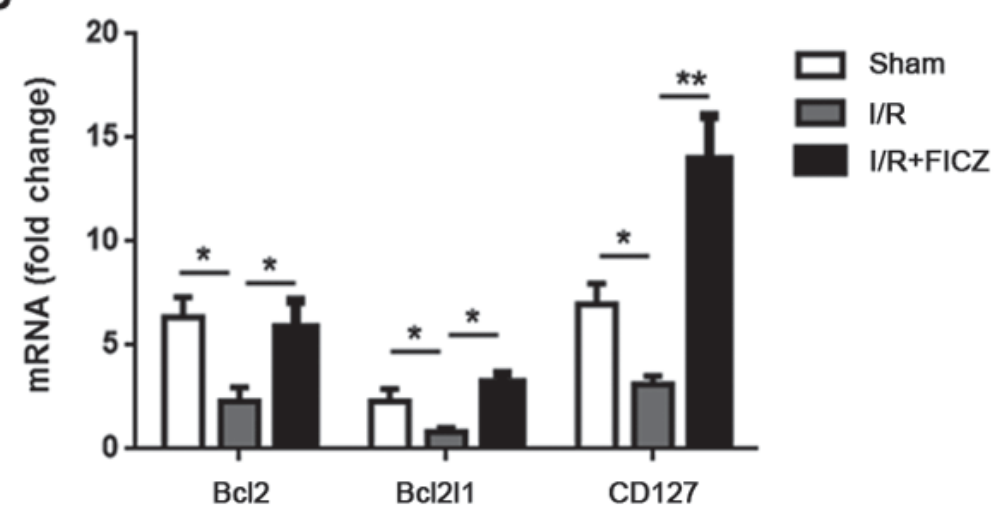

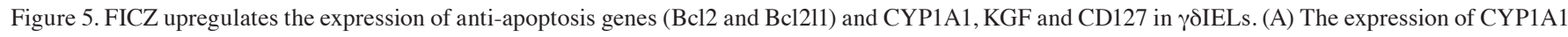
and KGF in $\gamma \delta$ ELs was analyzed by qPCR. (B) The expression of Bcl2, Bcl211, and CD127 mRNA in $\gamma \delta$ IELs was analyzed by qPCR. Each group had 2-3 mice and every experiment was repeated three times. ${ }^{*} \mathrm{P}<0.05$; ${ }^{* *} \mathrm{P}<0.01$. FICZ, 6-formylindolo(3,2-b)carbazole; IELs, intraepithelial lymphocytes; Bcl2, B-cell lymphoma 2; Bcl211, BCL2-like 1; KGF, keratinocyte growth factor; CYP1A1, cytochrome P450 1A1; CD127, cluster of differentiation 127.

has been widely established that functional activation of the AhR causes nuclear translocation, which results in the transcriptional activation of phase I and II detoxification enzymes, such as CYP1A1. Therefore, we determined the functional activation of the AhR by analyzing the expression of CYP1A1 mRNA in $\gamma \delta I E L s$.

In summary, it was demonstrated that intestinal I/R injury results in a decrease in CD127 on the membrane of small intestinal $\gamma \delta$ IELs, which is accompanied by apoptosis. FICZ administration enhanced CD127 expression and the $\gamma \delta I E L$ subset and downregulated inflammatory mediator genes expressed by IELs. Together these results indicate that FICZ administration after intestinal I/R attenuates apoptosis of $\gamma \delta$ IELs and maintains the small intestinal $\gamma \delta$ IEL population, as well as reducing the expression of intestinal inflammatory cytokines, which probably ameliorates the severity of IR-induced injury to the small intestinal barrier.

\section{Acknowledgements}

Not applicable.

\section{Funding}

This study was supported by grants from the National Natural Science Foundation of China (NSFC 81330013 to HY, NSFC
81501661 to MY, NSFC 81770524 and 81470803 to WX and NSFC 81370054 to YC) and the Innovative Research Team of Ministry of Education of China (IRT_17R16 to HY).

\section{Availability of data and materials}

All datasets used in the current study are available from the corresponding author upon reasonable request.

\section{Authors' contributions}

HY and WX conceived and designed the study. ZZ, AP, MY and LS performed the experiments. ZZ and AP collected the data and YC performed the data analysis. ZZ, AP and YC wrote the manuscript. All authors have read and approved the final manuscript and agree to be accountable for all aspects of the work in ensuring that questions related to the accuracy or integrity of any part of the work are appropriately investigated and resolved.

\section{Ethics approval and consent to participate}

All procedures performed with the mice were according to the Guidelines for the Care and Use of Laboratory Animals, as set forth and approved by the University Committee at the Army Medical University, Chongqing, China. 


\section{Patient consent for publication}

Not applicable.

\section{Competing interests}

The authors declare that they have no competing interests.

\section{References}

1. Gennaro M, Ascer E, Matano R, Jacobowitz IJ, Cunningham JN $\mathrm{Jr}$ and Uceda P: Acute mesenteric ischemia after cardiopulmonary bypass. Am J Surg 166: 231-236, 1993.

2. Guan YF, Pritts TA and Montrose MH: Ischemic post-conditioning to counteract intestinal ischemia/reperfusion injury. World J Gastrointest Pathophysiol 1: 137-143, 2010.

3. Nguyen LP and Bradfield CA: The search for endogenous activators of the aryl hydrocarbon receptor. Chem Res Toxicol 21 102-116, 2008

4. Kewley RJ, Whitelaw ML and Chapman-Smith A: The mammalian basic helix-loop-helix/PAS family of transcriptional regulators. Int J Biochem Cell Boil 36: 189-204, 2004.

5. Schrenk D: Impact of dioxin-type induction of drug-metabolizing enzymes on the metabolism of endo- and xenobiotics. Biochem Pharmacol 55: 1155-1162, 1998.

6. Esser C: Biology and function of the aryl hydrocarbon receptor: Report of an international and interdisciplinary conference. Arch Toxicol 86: 1323-1329, 2012.

7. Lahoti TS, Boyer JA, Kusnadi A, Muku GE, Murray IA and Perdew GH: Aryl hydrocarbon receptor activation synergistically induces lipopolysaccharide-mediated expression of proinflammatory chemokine (c-c motif) ligand 20. Toxicol Sci 148 : 229-240, 2015

8. Gras D, Chanez P, Vachier I, Petit A and Bourdin A: Bronchial epithelium as a target for innovative treatments in asthma. Pharmacol Ther 140: 290-305, 2013

9. Zimmerman BJ and Granger DN: Mechanisms of reperfusion injury. Am J Med Sci 307: 284-292, 1994.

10. Mallick IH, Yang W, Winslet MC and Seifalian AM Ischemia-reperfusion injury of the intestine and protective strategies against injury. Dig Dis Sci 49: 1359-1377, 2004.

11. Ye Y, Yue M, Jin X, Chen S and Li Y: Isolation of murine small intestinal intraepithelial gammadeltat cells. Immunol Invest 39: 661-673, 2010

12. Kuhl AA, Pawlowski NN, Grollich K, Loddenkemper C, Zeitz M and Hoffmann JC: Aggravation of intestinal inflammation by depletion/deficiency of gammadelta $\mathrm{T}$ cells in different types of IBD animal models. J Leukoc Boil 81: 168-175, 2007.

13. Inagaki-Ohara K, Chinen T, Matsuzaki G, Sasaki A, Sakamoto Y, Hiromatsu K, Nakamura-Uchiyama F, Nawa Y and Yoshimura A: Mucosal T cells bearing TCRgammadelta play a protective role in intestinal inflammation. J Immunol 173: 1390-1398, 2004.

14. Yang H, Antony PA, Wildhaber BE and Teitelbaum DH Intestinal intraepithelial lymphocyte gamma delta-T cell-derived keratinocyte growth factor modulates epithelial growth in the mouse. J Immunol 172: 4151-4158, 2004.

15. Ismail AS, Behrendt CL and Hooper LV: Reciprocal interactions between commensal bacteria and gamma delta intraepithelial lymphocytes during mucosal injury. J Immunol 182: 3047-3054, 2009 .

16. Qiu Y, Yu M, Yang Y, Sheng H, Wang W, Sun L, Chen G, Liu Y, Xiao W and Yang H: Disturbance of intraepithelial lymphocytes in a murine model of acute intestinal ischemia/reperfusion. J Mol Histol 45: 217-227, 2014.

17. Ji T, Xu C, Sun L, Yu M, Peng K, Qiu Y, Xiao W and Yang H: Aryl hydrocarbon receptor activation down-regulates IL-7 and reduces inflammation in a mouse model of DSS-induced colitis. Dig Dis Sci 60: 1958-1966, 2015

18. Li Y, Innocentin S, Withers DR, Roberts NA, Gallagher AR, Grigorieva EF, Wilhelm C and Veldhoen M: Exogenous stimuli maintain intraepithelial lymphocytes via aryl hydrocarbon receptor activation. Cell 147: 629-640, 2011.
19. Livak KJ and Schmittgen TD: Analysis of relative gene expression data using real-time quantitative PCR and the 2(-Delta Delta C(T)) method. Methods 25: 402-8, 2001

20. Vajdovich P: Free radicals and antioxidants in inflammatory processes and ischemia-reperfusion injury. Vet Clin North Am Small Anim Pract 38: 31-123, 2008.

21. Edrees WK, Lau LL, Young IS, Smye MG, Gardiner KR, Lee B, Hannon RJ and Soong CV: The effect of lower limb ischaemia-reperfusion on intestinal permeability and the systemic inflammatory response. Eur J Vasc Endovasc Surg 25: 330-335, 2003.

22. Kannan KB, Colorado I, Reino D, Palange D, Lu Q, Qin X, Abungu B, Watkins A, Caputo FJ, Xu DZ, et al: Hypoxia-inducible factor plays a gut-injurious role in intestinal ischemia reperfusion injury. Am J Physiol Gastrointest Liver Physiol 300: G853-G861, 2001.

23. Vidali F, Di Sabatino A, Broglia F, Cazzola P, Biancheri P, Viera FT, Vanoli A, Alvisi C, Perego M and Corazza GR: Increased CD8+ intraepithelial lymphocyte infiltration and reduced surface area to volume ratio in the duodenum of patients with ulcerative colitis. Scand J Gastroenterol 45: 684-689, 2010.

24. Lindsey WB, Lowdell MW, Marti GE, Abbasi F, Zenger V, King KM and Lamb LS Jr: CD69 expression as an index of T-cell function: Assay standardization, validation and use in monitoring immune recovery. Cytotherapy 9: 123-132, 2007.

25. Montufar-Solis D, Garza T and Klein JR: T-cell activation in the intestinal mucosa. Immunol Rev 215: 189-201, 2007.

26. Ekong UD, Luo X, Yu M, Wang D, Miller SD and O'Gorman MR Lymphocyte activation markers may predict the presence of donor specific alloreactivity in pediatric living related liver transplant recipients. Hum Immunol 72: 392-397, 2011.

27. Boismenu R and Havran WL: Modulation of epithelial cell growth by intraepithelial gamma delta T cells. Science 266: 1253-1255, 1994.

28. Schluns KS and Lefrancois L: Cytokine control of memory T-cell development and survival. Nat Rev Immunol 3: 269-279, 2003.

29. Maki K, Sunaga S, Komagata Y, Kodaira Y, Mabuchi A, Karasuyama H, Yokomuro K, Miyazaki JI and Ikuta K: Interleukin 7 receptor-deficient mice lack gammadelta T cells. Proc Natl Acad Sci USA 93: 7172-7177, 1996.

30. Cai YJ, Wang WS, Liang HY, Sun LH, Teitelbaum DH and Yang H: Keratinocyte growth factor up-regulates Interleukin-7 expression following intestinal ischemia/reperfusion in vitro and in vivo. Int J Clin Exp Pathol 5: 569-580, 2012.

31. Weitkamp JH, Rosen MJ, Zhao Z, Koyama T, Geem D, Denning TL, Rock MT, Moore DJ, Halpern MD, Matta P and Denning PW: Small intestinal intraepithelial TCRgammadelta+ $\mathrm{T}$ lymphocytes are present in the premature intestine but selectively reduced in surgical necrotizing enterocolitis. PloS One 9: e99042, 2014

32. Haas E, Rutgen BC, Gerner W, Richter B, Tichy A, Galler A, Bilek A, Thalhammer JG, Saalmüller A and Luckschander-Zeller N: Phenotypic characterization of canine intestinal intraepithelial lymphocytes in dogs with inflammatory bowel disease. J Vet Intern Med 28: 1708-1715, 2014.

33. Lee WY, Hu YM, Ko TL, Yeh SL and Yeh CL: Glutamine modulates sepsis-induced changes to intestinal intraepithelial gammadeltaT lymphocyte expression in mice. Shock 38: 288-293, 2012.

34. Khoshnan A, Tindell C, Laux I, Bae D, Bennett B and Nel AE: The NF-kappa B cascade is important in Bcl-xL expression and for the anti-apoptotic effects of the CD28 receptor in primary human CD4+ lymphocytes. J Immunol 165: 1743-1754, 2000

35. Cai Y, Wang W, Liang H, Sun L, Teitelbaum DH and Yang H: Keratinocyte growth factor improves epithelial structure and function in a mouse model of intestinal ischemia/reperfusion. PloS One 7: e44772, 2012

36. Maretta M, Toth S, Bujdos M, Toth S Jr, Jonecova Z and Vesela J: Alterations of epithelial layer after ischemic preconditioning of small intestine in rats. J Mol Histol 43: 171-178, 2012.

37. Cerqueira NF, Hussni CA and Yoshida WB: Pathophysiology of mesenteric ischemia/reperfusion: A review. Acta Cir Bras 20: 336-343, 2005. 